\title{
Exploitation of renewable resources with differentiated technologies: an evolutionary analysis
}

\author{
F. Lamantia ${ }^{1, a}$, D. Radi ${ }^{1, b, *}$ \\ ${ }^{a}$ Department of Economics, Statistics and Finance, University of Calabria, Italy \\ ${ }^{b}$ Department of Management, Economics and Quantitative Methods, University of Bergamo, Italy
}

\begin{abstract}
In this paper we propose a dynamical model of technology adoption for the exploitation of a renewable natural resource. Each technology has a different efficiency and environmental impact. The process of technology adoption over time is modeled through an evolutionary game employed by profit maximizing exploiters. The loss in profits due to lower efficiency levels of environmentallyfriendly technologies can be counterbalanced by the higher consumers' propensity to pay for greener goods. The dynamics of the resource take place in continuous time, whereas the technology choice can be revised either in continuous-time or in discrete-time. In the latter case, the model assumes the form of a hybrid system, whose dynamics is mainly explored numerically. We shows that: 1) overexploitation of the resource arises whenever the reduction in harvesting due to a lower efficiency of clean technology is more than compensated by a higher propensity to pay for greener goods; 2) the difference between the fixed costs of these technologies can be exogenously fixed to provide an incentive for adopting clean technology without affecting the long-run level of the resource; and 3) in some cases, discrete switching of the technology causes overshooting in the dynamics whereas in others it enhances the stability of the system.
\end{abstract}

Keywords: Resource dynamics, Stock externality, Evolutionary game theory, Hybrid systems.

\section{Introduction}

A main issue in the exploitation of common property resources is the so-called "stock externality": individual exploiters do not take into account the effects of their current catch on the resource

* Corresponding author

Email addresses: lamantia@unical.it (F. Lamantia), davide.radi@unibg.it (D. Radi) 
and on its future abundance. The non-coincidence between individual optima and collective optima is commonly referred to as the "tragedy of the commons", after [21, and characterizes the exploitation of almost all shared natural resources, see also [13] and 28. Moreover, enforcing control on the resource is a very difficult and often an ineffective task.

In this paper we address a descriptive model for the exploitation of a common pool renewable resource, on which the regulator does not enforce any restraint. However, some exploiters can decide to employ a less efficient but more "environmentally-friendly" technology if the loss in efficiency is counterbalanced by a higher price that consumers might be willing to pay for the greener product 1 The choice of the technology, which is an exogenous component of the mode 2 , only depends on the agents' assessment on expected profits and not on ethical or environmental concerns, as agents are assumed to be selfish profit maximizers. Exploiters have to make two choices over time: which technology to adopt and, given that, the quantity to harvest. With respect to the problem of technology adoption, we model it through an evolutionary game in the spirit of [24] and [34], as is customary in natural resource exploitation models, see, among others, [32], 36, [1, [8, [9]. Thus, agents can switch from the selected technology to another that is available if they expect that the change can be profitable. Regarding the quantities to be harvested, we follow [32] and assume that agents choose their catches continuously in order to be in a Nash equilibrium at any given time.

We first address the case in which the choice of the technology can be revised continuously. This step constitutes a useful benchmark to understand the main qualitative properties of the model. Then we break down this assumption to conceive a scenario that is more similar to what could take place in a more realistic setting. In fact, changing technology immediately is not feasible in practical cases for different reasons, the most obvious of which is due to the interval of time required to conclude a single harvesting operation. Thus, it is natural to assume that only after a certain time interval may a change in the employed technology take place. In this circumstance the system can be modeled mathematically through a hybrid model, including continuous-time resource growth and impulsive changes of strategies. The latter takes place at discrete points in time according to an evolutionary endogenous switching mechanism. In recent years, hybrid dynamical systems have

\footnotetext{
${ }^{1}$ Empirical evidence has shown that the introduction of an eco-label can indeed change market behavior, see 33 for the case study of the dolphin-safe labeling.

${ }^{2}$ For overviews about the existence and the importance of heterogeneity among economic agents with respect to technology adoption, product activities and evolutionary paradigms for the diffusion of technologies, we refer the reader to [14]. For an example of technological adoption processes in fisheries, see [15].
} 
been widely employed for studying real-world problems in several branches of applied mathematics, such as engineering, biology and biomedical science (see e.g. [11, [12, 3], 18, [19, [20]). In fishery models, hybrid systems have been recently proposed in [6] and [7].

The first goal of this paper is to gain an understanding of the influence of the length of the switching interval on the dynamics of the natural resource and profits. In some cases, continuous technology switching just speeds up the convergence to the same attractor of the hybrid system. In others, discrete and continuous switchings exhibit different long-run behaviors. Interestingly, under some circumstances discrete switching may even introduce a stabilizing effect in the model because of more inertia in the system when switching decisions are based upon past profits. Another aim of the paper is to assess whether an unregulated use of the resource can be sustained in the long-run. Although this is true in some cases, we show some examples where every agent tends to use the less-efficient technology but the level of the resource in the long run is lower than the level obtained if every agent would have used the traditional technology. This occurs whenever the high price for the green product induces too many exploiters to over-harvest it. In these cases, the market itself is not able to mitigate the effects of the tragedy of the commons but additional regulatory policies must be introduced. For instance, a regulator could avoid poverty traps by providing an incentive for adopting a technology over the other. Analytic and numerical analysis show that the stability of equilibria is quite sensible to the difference in the fixed costs between the two available technologies but the level of harvesting is not affected by this difference. As a result, a regulator can employ the difference in fixed costs to steer the system towards the preferred long-run level of the resource.

The problem of adopting a less efficient but more environmentally-friendly technology is motivated by some real-world cases occurring in fisheries outside exclusive economic zones. In this respect, a well-known example regards the landing of yellowfin tuna and the marketing of "dolphin safe" labels? Dolphins commonly swim together with tunas, but closer to the surface. Therefore fishing boats spot dolphins more easily than tuna. Consequently, although dolphins are a non-target species and have no commercial value, they have been largely captured as bycatch in tuna fisheries. Netting dolphins with tunas has severely endangered the population of dolphins. This issue motivated the introduction in the late 20th century of the "dolphin safe" labels in several countries

\footnotetext{
${ }^{3}$ See the 'Dolphin Protection Consumer Information Act' at http://dolphinsafe.gov/dolprot.pdf
} 
such as the U.S., the U.K. or New Zealand 4 . The presence of bycatch-free labels created market segmentation with different prices for labeled and non-labeled tuna cans. The model proposed in this paper can be regarded as a stylized version of this problem, but with appropriate adjustments it can be easily adapted to describe other real-world cases.

This paper is structured as follows. Section 2 introduces the bioeconomic model. Section 3 is devoted to the analysis of the model with evolutionary switching of harvesting technologies in continuous-time and introduces a formulation of the model with continuous-time growth of the resource and discrete switching of technologies. Section 4 proposes several numerical analysis comparing the (transient and long-run) dynamic properties for the two models, also briefly addressing some policy measures to avoid poverty traps and stimulate the adoption of the environmentallyfriendly technology. Section 5 concludes.

\section{Bioeconomic Model}

As is customary in bioeconomic models, we assume that the target resource (e.g. yellowfin tuna) follows a logistic differential equation subjected to harvesting 5

$$
\dot{z}=z(\alpha-\beta z)-H(z)
$$

where $\alpha$ is the intrinsic rate of growth, $\alpha / \beta$ is the natural carrying capacity and $H(z)$ denotes the instantaneous total harvest (or harvesting rate) by the $N$ agents who own the common pool. Below we detail the functional form of the total harvest $H(z)$.

Two exogenous technologies are available for catching the target species which we label $s$ ("standard") and $c$ ("clean") in the following. Technology $s$ is more efficient but less environmentally friendly than $c$. The different efficiencies are reflected in the different catchability coefficients $q_{s}$ and $q_{c}$ with $q_{s}>q_{c}$. At any time period, the $N$ harvesters can be split in two groups according to the technology they use, with $n_{c}$ "clean" agents and $n_{s}=N-n_{c}$ "standard" agents.

\footnotetext{
${ }^{4}$ For more details on this specific issue we refer to 33 .

${ }^{5}$ Since no confusion arises, in this Section we write $\dot{z}(t)=\dot{z}$ and $z(t)=z$ to simplify the notation.

${ }^{6}$ In particular, exogenous technologies can be related to different production practices. Even though the employed equipment is the same for the two technologies, the difference between a bycatch-free production practice and a standard one is the choice of the fishing site. In terms of costs, this difference reflects to the distance between different sites and the amount of fuel required. In terms of production efficiency, the amount of harvested resources for a unit of time decreases. All these effects are captured by parameters $q_{i}$.
} 
Another standard assumption in similar models is that the catch of the target species can be described by a Cobb-Douglas harvesting function with constant returns to scale7. Thus, a fisherman employing technology $i \in\{s, c\}$ will catch the quantity:

$$
h_{i}(z)=\sqrt{q_{i} E_{i} z}
$$

where $E_{i}$ denotes his/her current harvesting effort. The total cost of fishing for a type $i$ agent, denoted by $C_{i}$, is proportional to the effort plus a fixed cost 8 , i.e., $C_{i}=c_{i}+\gamma E_{i}$. In terms of harvested quantity, the total cost is

$$
C_{i}(h(z))=c_{i}+\gamma \frac{(h(z))^{2}}{q_{i} z}, i \in\{s, c\} .
$$

Notice that marginal cost for "clean" harvesting is higher than for standard harvesting. Current aggregate harvest $H(z)=n_{c} h_{c}(z)+n_{s} h_{s}(z)$ is wholly supplied to the market. In the following we denote by $a_{i}$ the (constant) price for a product obtained through technology $i$. Given the interpretation of the model, below we always assume that $a_{c}>a_{s}$, as consumers might be willing to pay more for the product obtained through a more environmentally-friendly technology. The assumption of a perfectly elastic demand for the resource is well justified whenever the resource is a staple food for the consumer or several substitutes to the resource are traded on the market, see more on this point $[13$.

Fishermen are myopic, in the sense that they will maximize their instantaneous profits but not the discounted flows of future profits. However, it is possible that they revise the decision on a chosen technology by considering its past performance, as detailed below. With respect to payoffs, a representative fisherman employing technology $i \in\{s, c\}$ maximizes the following profit at any time period

$$
\pi_{i}(z)=p_{i} h_{i}(z)-c_{i}-\gamma \frac{\left(h_{i}(z)\right)^{2}}{q_{i} z}
$$

through the first order condition $\frac{\partial \pi_{i}}{\partial h_{i}}=0$, which is also sufficient being $\frac{\partial^{2} \pi_{i}}{\partial h_{i}^{2}}=-\frac{2 \gamma}{q_{i} z}<0$.

Thus, employing symmetry among all players with the same technology, the Nash Equilibrium

\footnotetext{
${ }^{7}$ The use of harvesting functions with constant returns to scale is common in resource economics, see 13 , for a detailed discussion on the topic. Other forms of harvesting functions are also employed, see e.g. [30.

${ }^{8}$ The difference in fixed costs between the two harvesting strategies can be related either to additional costs for the labeling of the environmentally-friendly product if $c_{c}>c_{s}$ or to tax relief if $c_{c}<c_{s}$. Given this interpretation, to some extent the difference in fixed costs could be regarded as a possible policy measure to the fishing authority.
} 
harvesting strategy for a type $i$ agent reads

$$
h_{i}^{N E}(z)=\frac{a_{i} q_{i} z}{2 \gamma} ; i \in\{s, c\}
$$

with $h_{i}^{N E}(z) \geq 0$ whenever $z \geq 0$.

Profits 3 at the Nash equilibrium 4 are nonnegative for sufficiently low fixed costs $c_{i}$, being $\pi_{i}^{*}(z)=\frac{a_{i}^{2} q_{i} z}{4 \gamma}-c_{i}$.

\section{Technology choice and replicator dynamics}

\subsection{Instantaneous Switching}

In this section, we explicitly formulate a dynamic mechanism for agents' switching between two available technologies. Agents tend to switch from one strategy to another if they expect this change to be profitable for them, according to the paradigms of evolutionary game theory. In general, a switching cost should be included in the model. However, for the specific problem under consideration here, we do not include such cost:9 The opportune changes in the model to include switching costs are outlined in Appendix A

We firstly developed the model with continuous-time switching, then we relax the assumption that agents are able to change their technology continuously and we introduce a time interval after which agents can switch to the other technology.

In the case of agents revising their strategy (i.e. the employed technology) continuously and instantaneously, we follow [32] and assume that agents harvest the Nash equilibrium quantity (4) at any given time period. The corresponding profits 3 evaluated at the Nash equilibrium, denoted by $\pi_{i}^{*}(z(t)), i \in\{s, c\}$, are taken as fitness measures for the adoption of each technology. Among the possible evolutionary dynamics, we focus on the most common one, namely the replicator in continuous-time (see 34 and 24]). Let us denote by $r(t)=\frac{n_{s}}{N}$ the fraction of agents using "standard" technology (and $1-r(t)$ the complementary fraction of agents using "clean" technology).

\footnotetext{
${ }^{9}$ Take, for instance, the case in which the environmentally-friendly technology consists in bycatch-free harvesting. Then the main difference between this kind of harvesting and a traditional one relies only on the employed searching methods for tuna, which do not require additional equipment. Whereas a traditional technology targets directly to dolphins for spotting tuna ('dolphin set' technology), the bycatch-free technology targets either schools of tuna ('school set' technology) or floating debris ('log set' technology). For all of these three searching technologies, the same equipment is required, i.e. crew with binoculars, speedboats, and helicopters that are launched off the purse seiner, see 23]. It follows that exploiters can decide to change the searching method in use at each fishing operation without additional costs.
} 
The replicator equation is one possible way to model that from $\pi_{s}^{*}(z(t))>\pi_{c}^{*}(z(t))$ it follows that $\dot{r}(t)>0$ and vice versa. Mathematically, the dynamical system with resource and replicator dynamics is given by the following system of ODEs

$$
\left\{\begin{array}{l}
\dot{z}(t)=z(t)(\alpha-\beta z(t))-N\left(r(t) h_{s}^{N E}(z(t))+(1-r(t)) h_{c}^{N E}(z(t))\right) \\
\dot{r}(t)=r(t)(1-r(t))\left[\pi_{s}^{*}(z(t))-\pi_{c}^{*}(z(t))\right]
\end{array}\right.
$$

\subsection{Equilibria and stability}

Here we analyze the steady states and their stability properties for the continuous-time replicator dynamics (5). The system of ODEs (5) can be rewritten as

$$
\left\{\begin{array}{l}
\dot{z}(t)=z(t)(\alpha-\beta z(t))-N\left[r(t) \frac{a_{s} q_{s}}{2 \gamma} z(t)+(1-r(t)) \frac{a_{c} q_{c}}{2 \gamma} z(t)\right] \\
\dot{r}(t)=r(t)(1-r(t))\left[\frac{a_{s}^{2} q_{s}-a_{c}^{2} q_{c}}{4 \gamma} z(t)+\xi\right]
\end{array}\right.
$$

where $\xi=c_{c}-c_{s}$. In the proposition below we denote by $\widehat{\xi}=\frac{\left(a_{c}^{2} q_{c}-a_{s}^{2} q_{s}\right)\left(2 \alpha \gamma-N a_{c} q_{c}\right)}{8 \beta \gamma^{2}}$, and $\bar{\xi}=$ $\frac{\left(a_{c}^{2} q_{c}-a_{s}^{2} q_{s}\right)\left(2 \alpha \gamma-N a_{s} q_{s}\right)}{8 \beta \gamma^{2}}$.

Proposition 1 (Equilibria and stability). The ODEs model (6) admits the following equilibria in the phase space $(z, r)$ :

- Equilibria with extinction of the resource:

$$
E_{0,0}=(0,0) \text { and } E_{0,1}=(0,1)
$$

with $E_{0,0}$ always unstable (saddle or node) for $\xi>0$ and either stable if $a_{c} q_{c}>\frac{2 \gamma \alpha}{N}$ or unstable (saddle) if $a_{c} q_{c}<\frac{2 \gamma \alpha}{N}$ for $\xi<0$ and $E_{0,1}$ always unstable (saddle or node) for $\xi<0$ and either stable if $a_{s} q_{s}>\frac{2 \gamma \alpha}{N}$ or unstable if $a_{s} q_{s}<\frac{2 \gamma \alpha}{N}$ for $\xi>0$.

- Boundary equilibria, each of which involving the employment of only one harvesting technology:

$$
\widehat{E}_{\widehat{z}, 0}=(\widehat{z}, 0)=\left(\frac{\alpha}{\beta}-\frac{N a_{c} q_{c}}{2 \beta \gamma}, 0\right) \text { and } \bar{E}_{\bar{z}, 1}=(\bar{z}, 1)=\left(\frac{\alpha}{\beta}-\frac{N a_{s} q_{s}}{2 \beta \gamma}, 1\right)
$$

Moreover:

- $\widehat{E}_{\widehat{z}, 0}$ is feasible (i.e. with positive biomass) iff $a_{c} q_{c}<\frac{2 \alpha \gamma}{N}$, i.e. iff $E_{0,0}$ is unstable along the $r$ axis.

$-\bar{E}_{\bar{z}, 1}$ is feasible iff $a_{s} q_{s}<\frac{2 \alpha \gamma}{N}$, i.e. if $E_{0,1}$ is unstable along the invariant line $r=1$. 
- $\widehat{E}_{\widehat{z}, 0}$ and $\bar{E}_{\bar{z}, 1}$ are both feasible if $\frac{2 \alpha \gamma}{N}>\max \left\{a_{c} q_{c}, a_{s} q_{s}\right\}$.

- With respect to the stability

* $\widehat{E}_{\widehat{z}, 0}$ is a stable node for $\xi<\widehat{\xi}$ or a saddle for $\xi>\widehat{\xi}$;

$* \bar{E}_{\bar{z}, 1}$ is a stable node for $\xi>\bar{\xi}$ or a saddle for $\xi<\bar{\xi}$.

- A unique equilibrium that involves the employment of both technologies:

$$
E^{*}=\left(z^{*}, r^{*}\right)=\left(\frac{4 \xi \gamma}{a_{c}^{2} q_{c}-a_{s}^{2} q_{s}}, \frac{a_{c} q_{c} N-2 \alpha \gamma+\frac{8 \xi \beta \gamma^{2}}{a_{c}^{2} q_{c}-a_{s}^{2} q_{s}}}{N\left(a_{c} q_{c}-a_{s} q_{s}\right)}\right)
$$

Moreover:

- if $a_{c} q_{c} \geq a_{s} q_{s}$ or if $a_{c} q_{c}<a_{s} q_{s}$ with $a_{c}^{2} q_{c}<a_{s}^{2} q_{s}$ then $E^{*}$ is feasible, i.e. $z^{*}>0$ and

$0<r^{*}<1$, provided that

$$
\widehat{\xi}<\xi<\bar{\xi}
$$

In this case $E^{*}$ is always a stable point (either spiral or node).

- if $a_{c} q_{c}<a_{s} q_{s}$ with $a_{c}^{2} q_{c}>a_{s}^{2} q_{s}$, then $E^{*}$ is feasible provided that

$$
\bar{\xi}<\xi<\widehat{\xi}
$$

In this case $E^{*}$ is always a saddle point.

- at $\xi=\widehat{\xi}$ it is $r^{*}=0$ and $E^{*}=\widehat{E}_{\widehat{z}, 0}$, whereas at $\xi=\bar{\xi}$ it is $r^{*}=1$ and $E^{*}=\bar{E}_{\bar{z}, 1}$.

Proof. See Appendix B

From this theorem it is possible to derive some preliminary bioeconomic policy implications that will be analyzed in more detail in the next section. First of all, it is worth noting that when there is a reduction in harvesting due to the lower efficiency of clean technology $q_{c}$ that is more than compensated by the propensity to pay for a greener product $a_{c}$, i.e. when $h_{c}^{N E}(z)>h_{s}^{N E}(z)$ or equivalently when $a_{c}>\frac{a_{s} q_{s}}{q_{c}}$, the border equilibria $\widehat{E}_{\widehat{z}, 0}$ and $\bar{E}_{\bar{z}, 1}$ are such that $\widehat{z}<\bar{z}$. In this case, even if agents endogenously select the equilibrium $\widehat{E}_{\widehat{z}, 0}$ in which only clean technology is used, the 
resource will be overexploited in the long-run. This problem can be overcome by controlling the propensity to pay for greener product, i.e. imposing that $a_{c}<\frac{a_{s} q_{s}}{q_{c}}$. Another interesting aspect of the theorem regards the role played on the stability of the equilibria by the difference in the fixed costs of the two technologies $\xi=c_{c}-c_{s}$. Let us consider for example the situation in which the two border equilibria $\widehat{E}_{\widehat{z}, 0}$ and $\bar{E}_{\bar{z}, 1}$ are both feasible and stable, so that $\bar{\xi}<\xi<\widehat{\xi}$. By subsidizing clean technology or taxing traditional technology, it is possible to reduce the difference in the fixed costs between the two technologies so that $\xi<\bar{\xi}<\widehat{\xi}$. As a result, the two border equilibria do not change their position in the state space but the equilibrium $\bar{E}_{\bar{z}, 1}$ becomes unstable and $\widehat{E}_{\widehat{z}, 0}$ is the only stable equilibrium. In other terms, clean technology prevails without reducing the stock size of the target resource.

In respect to the inner equilibrium $E^{*}$, Proposition 1 states that an equilibrium in which both technologies are used can exist only if the difference in the fixed costs of the two technologies $\xi$ is sufficiently low. Provided that this happens, such an equilibrium is stable in the following cases: (i) when an individual catch with clean technology is not lower than the corresponding intake with standard technology (i.e. whenever $a_{c} q_{c} \geq a_{s} q_{s}$ ) or when (ii) individual profits without fixed costs associated to "environmentally-friendly" technology are lower than the corresponding profits obtained with the standard technology $\left(a_{c}^{2} q_{c}<a_{s}^{2} q_{s}\right)$. On the other hand, if any agent employing technology $c$ harvests less than any agent using technology $s$ (i.e. $a_{c} q_{c}<a_{s} q_{s}$ ), then an inner equilibrium is feasible if the profits (without fixed costs) to the first agent are higher than the profits to the second $\left(a_{c}^{2} q_{c}>a_{s}^{2} q_{s}\right)$. However, such an equilibrium is always a saddle point for the evolutionary dynamics of the system. Finally, note that the inner equilibrium $E^{*}$ cannot be stable when the border equilibria $\widehat{E}_{\widehat{z}, 0}$ and $\bar{E}_{\bar{z}, 1}$ are stable and vice versa.

For the various stability conditions of the border equilibria a clear economic interpretation can be given. For example, the local stability condition for $\widehat{E}_{\widehat{z}, 0}$, i.e., $\xi<\widehat{\xi}$, can be rewritten as $\pi_{c}^{*}(\widehat{z})>\pi_{s}^{*}(\widehat{z})$, which has a straightforward meaning: the equilibrium in which all the fishermen use the environmentally-friendly technology is stable if this technology outperforms the alternative strategy in a neighborhood of the equilibrium. Similar and opposite considerations hold true for the equilibrium $\bar{E}_{\bar{z}, 1}$.

\subsection{Discrete-time Switching}

Up to now, we have considered the evolutionary game in which agents decide instantaneously and continuously on which technology to adopt. In practice, it would be very difficult for the 
exploiters to realize such an instantaneous switching, for the reasons outlined in the introduction. In this section we formulate the model assuming that a minimum time interval $\Delta l$ exists after which switching may take place. The time interval $\Delta l$ can be interpreted as the time of a single fishing operation ${ }^{10}$ For the sake of simplicity, we assume that the single fishing operation takes the same amount of time for both technologies.

Thus, at the end of each time period of length $\Delta l$, a representative agent employing technology $i \in\{s, c\}$ measures $\bar{\pi}_{i}^{*}(z(t))$, the net performance of his/her current harvesting strategy, as the exponentially decaying weighted average of historical profits over a time interval $[t-\Delta t, t]$, i.e.

$$
\bar{\pi}_{i}^{*}(z(t))=\frac{\delta}{1-e^{-\delta \Delta t}} \int_{t-\Delta t}^{t} e^{-\delta(t-\tau)} \pi_{i}^{N E}(z(\tau)) d \tau \quad ; i \in\{s, c\}
$$

where time delay $\Delta t \in(0, \infty)$ represents the profit history used to calculate the average past profits of the two harvesting strategies, and $\delta>0$ is a decay rate, assumed equal to all agents (see [22] for the same form of trend dynamics in an asset model). To make things easier, in this paper, we just consider the case $\Delta t=\Delta l$ and we do not make distinction between the tw11

The magnitude of $\bar{\pi}_{i}^{*}(z(t))$ is a fitness measure of playing strategy $i$ (see 34]). Again letting $r(t)=\frac{n_{s}}{N}$, the replicator dynamics can be expressed by a continuous-time growth equation for the biomass and a discrete (or pulse) strategy switching (a discrete decision-driven time). Under a synchronous updating of technologies (see [26]) the model can be written as

$$
\left\{\begin{array}{l}
\dot{z}(t)=z(t)(\alpha-\beta z(t))-N\left(r(t) h_{s}^{N E}(z(t))+(1-r(t)) h_{c}^{N E}(z(t))\right) \\
r(t)=\left\{\begin{array}{cc}
r(t-\Delta l) \frac{e^{\theta \bar{\pi}_{s}^{*}(z(t))}}{r(t-\Delta l) e^{\theta \bar{\pi}_{s}^{*}(z(t))}+(1-r(t-\Delta l)) e^{\theta \bar{\pi}_{c}^{*}(z(t))}} & \text { if } \frac{t}{\Delta l}=\left\lfloor\frac{t}{\Delta l}\right\rfloor \\
r\left(\left\lfloor\frac{t}{\Delta l}\right\rfloor \Delta l\right) & \text { otherwise }
\end{array}\right.
\end{array}\right.
$$

where $\lfloor x\rfloor$ is the largest integer not greater than $x$ (i.e. the floor of $x$ ), and $h_{i}^{N E}(z(t)), \bar{\pi}_{i}^{*}(z(t))$, $i=1,2$ are given, respectively, in (4) and (9).

The dynamical model in 10 is a hybrid system, because it combines the population growth model (1), which takes place in continuous-time, with a difference equation for the fraction of agents employing the two technologies. The two dynamics have different time scales and their

\footnotetext{
${ }^{10}$ For example, in the case of midwater trawl fishing technique, usually employed in tuna harvesting, $\Delta l$ can be interpreted as the amount of elapsed time from the moment of plunging the nets into the water until the fish is downloaded on the dock.

${ }^{11}$ Numerical simulations not reported here show that the dynamics of the model do not change substantially for $\Delta t \approx \Delta l$.
} 
modeling is best obtained through a hybrid model. Notice that the second equation in 10 is a standard replicator dynamic in discrete time. The monotone transformation $\bar{\pi}_{i}^{*}(z(t)) \rightarrow e^{\theta \bar{\pi}_{i}^{*}(z(t))}$ is employed for obtaining fitness measures with strictly positive values (see [34] and [25] for details). The parameter $\theta \in[0,+\infty)$, known as the intensity of choice, can be interpreted as a switching propensity.

\section{Numerical Analysis and Bioeconomic implications}

In this section we investigate some dynamic properties of the hybrid model (10) considering system (6) as a benchmark. Through these examples, we also underline the principal bioeconomic implications related in the use of clean technology. Before beginning the Section, we would like to remark that all the equilibria of model (6) are also equilibria of model (10), although the contrary is not necessarily true. Furthermore, the stability properties of equilibria can be different in the two versions of the model, namely with switching in continuous or discrete time, as highlighted below in the numerical simulations. In all the following examples we set an interval of length $\Delta l=1$, which can be regarded as the duration of a single harvesting operation, and a decay rate $\delta=0.01$ in 9 .

According to Proposition 1, the two border equilibria $\widehat{E}_{\widehat{z}, 0}$ and $\bar{E}_{\bar{z}, 1}$ are both feasible and stable for $\frac{2 \alpha \gamma}{N}>\max \left\{a_{c} q_{c}, a_{s} q_{s}\right\}$ and $\bar{\xi}<\xi<\widehat{\xi}$. Such a case is depicted in Figure (1), Panel (a). In particular, Panel (a) of Figure (1) shows a phase portrait in the plane $(z, r)$ for the continuous-time replicator model (6) with parameters: $\alpha=19, \beta=0.15, a_{s}=1, a_{c}=1.45, c_{s}=0.4, c_{c}=3$, $N=10, q_{s}=1.8, q_{c}=1, \gamma=0.5$. Under these parameters, in addition to the equilibria $\widehat{E}_{\widehat{z}, 0}$ and $\bar{E}_{\bar{z}, 1}$, model (6) admits two saddle equilibria $E_{0,1}$ and $E^{*}$ and the unstable node $E_{0,0}$.

Considering the system as in Figure (1), Panel (a) characterized by the two stable border equilibria $\widehat{E}_{\widehat{z}, 0}$ and $E_{\widetilde{z}, 1}$, Figures $[2$ and $(3)$ show the different trajectories between the continuous system (6) and the hybrid counterpart 10 for a different initial condition (i.c.). In details, with i.c. $(17,0.58)$, Figure 2 shows a trajectory converging to the border equilibrium $\bar{E}_{\bar{z}, 1}$, whereas with i.c. $(17,0.54)$, a trajectory converging to the stable node $\widehat{E}_{\widehat{z}, 0}$ is plotted in Figure 3 . In this example, the model with a continuous replicator and the one with a discrete replicator have qualitatively the same long-run dynamics, i.e. the same attractors with the same stability properties. The main difference between them concerns the different transitory dynamics toward the two stable equilibria $\widehat{E}_{\widehat{z}, 0}$ and $\bar{E}_{\bar{z}, 1}$, as clearly visible in the versus-time dynamics of Figures $(2)$ and $(3)$. In 

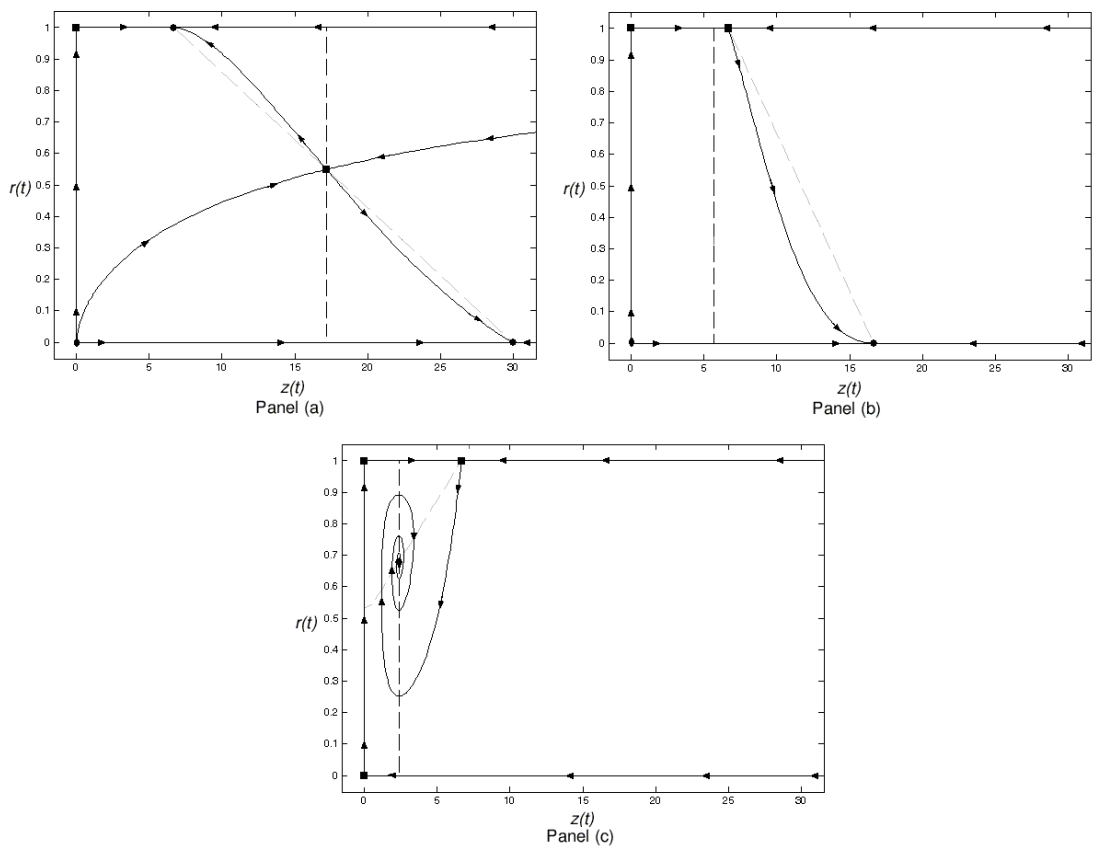

Figure 1: State spaces of the dynamical system (6). Panel (a): $\alpha=19, \beta=0.15, a_{s}=1, a_{c}=1.45, c_{s}=0.4, c_{c}=3$, $N=10, q_{s}=1.8, q_{c}=1, \gamma=0.5$; Panel (b): as in Panel (a) but $a_{c}=1.65$; Panel (c) as in Panel (a) but $a_{c}=2$. Black squares, circles and ovals represent, respectively, saddle points, stable nodes and unstable nodes. Dashed lines are the isoclines $\dot{z}=0$ (gray) and $\dot{r}=0$ (black). The black lines represent stable and unstable manifolds of the saddle points. The arrows indicate the direction of the flow of the dynamical system along the manifolds. 
the latter figures, the chosen initial conditions are close to the unstable manifold of the saddle $E^{*}$, separating the two basins of attraction of equilibria $\widehat{E}_{\bar{z}, 0}$ and $\bar{E}_{\bar{z}, 1}$ for the model with a continuous replicator. In general, this border of the two basins can be very different in the two versions of the model (continuous and discrete replicator), although in this specific example of Figures (2) and (3), numerical simulations not reported here confirm that the basins of attraction are similar for the two models.

As mentioned above, in this example the transitory dynamics of the two models is quite different. From Figure (2), Panels (b), (d) and (e), it is possible to notice that the hybrid model ensures higher levels of biomass, harvesting and profits along the entire trajectory converging to the border equilibrium $\bar{E}_{\bar{z}, 1}$ than the corresponding values in the continuous-time case. This seems to be counter-intuitive for profit-driven fishermen: intuitively, the faster they are able to adjust their profits toward the most performing strategy, the better their total profits should be. However, in this example along the entire trajectory it is $\dot{r} \geq 0$ (the fraction of agents employing technology $s$ increases over time) with fishermen moving towards an equilibrium with lower biomass (see Figure (2), Panel (b)). This is due to the fact that fishermen are attracted to strategy $s$ since it dominates strategy $c$ in all instances; but being myopic, fishermen do not consider the effect of their current harvesting on the future level of the resource (the "stock externality"). As a consequence, they tend to over-harvest by employing strategy $s$ and thus they end up with a profit that is lower than the one they could have obtained by always applying strategy $c$. More specifically, if the initial condition is in the basin of attraction of the equilibrium $\bar{E}_{\bar{z}, 1}$, then agents are "locked-in" in a prisoners' dilemma trap, where strategy $s$ always dominates strategy $c$ so that the strategy being played in the long run is $s$. This is clearly visible in Figure (2), Panel (e), where $\pi_{s}(t)$ (dashed-lines) is greater than or equal to $\pi_{c}(t)$ (continuous-lines) along the entire trajectories, thus explaining $\dot{r} \geq 0$. At the same time, both $\pi_{s}(t)$ and $\pi_{c}(t)$ decrease along the trajectory due to overfishing that depletes the biomass levels. Overall, myopic fishermen are faced with degenerating conditions. In this case, the equilibrium $\widehat{E}_{\widehat{z}, 0}$ would ensure a higher economic welfare, as visible in Figure 3 , Panel (e). Examples of similar poverty traps in related models are provided in [2], 10] and [5].

If otherwise, the initial condition is in the basin of attraction of the equilibrium $\widehat{E}_{\widehat{z}, 0}$, profitdriven agents move towards profits improvements. In fact, profits, harvesting and resource stock all increase along the trajectory until they reach the equilibrium $\widehat{E}_{\bar{z}, 0}$, where all agents employ the environmentally-friendly technology $c$. In this example, the replicator in continuous-time offers a 

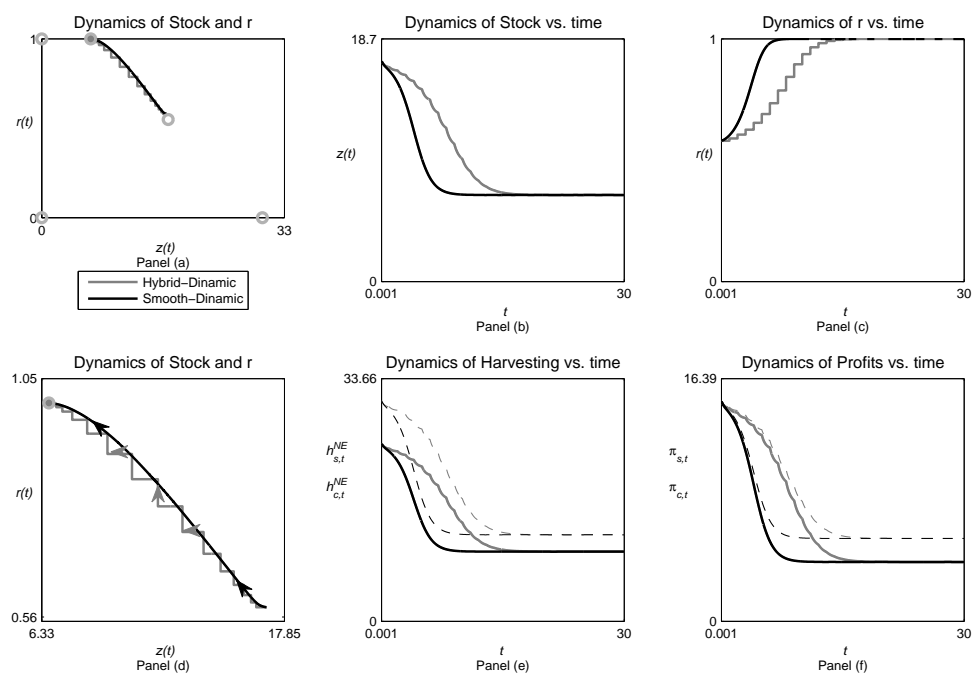

Figure 2: Dynamics of the model with continuous switchings (6) (black lines) and discrete switchings 10 (gray lines) with parameter values as in Fig. (1), Panel (a), and with $\delta=0.01, \theta=0.5$ and i.c. $(17,0.58)$. Panel (a) trajectories on the state space $(z, r)$; the gray and black dots represent the state of the hybrid and non-hybrid model respectively at time $t_{\text {end }}$. Panel (b) versus-time dynamics of biomass. Panel (c) versus-time dynamics of the fractions of agents using strategy $s$. Panel (d) enlargement of the dynamics in panel (a). Panel (e) versus-time dynamics of harvesting: dashed lines for strategy $s$, solid lines for strategy $c$. Panel (f) versus-time dynamics of profits: dashed lines for strategy $s$, solid lines for strategy $c$.

better economic performance along the transient trajectory than that in discrete-time. This is due to the fact that it moves faster to the equilibrium $\widehat{E}_{\widehat{z}, 0}$. From the point of view of a regulator ${ }^{12}$ trying to maximize the total industry profits over time, it is possible to show that in the examples of Figures (2) and (3) the singular state obtained through (C.6) is not accessible and the optimal control $\sqrt{\text { C.5 }}$ consists precisely in setting $r=0$ thus reaching the fixed point $\widehat{E}_{\widehat{z}, 0}$. This case occurs whenever the initial condition is taken in the basin of attraction of the equilibrium $\widehat{E}_{\widehat{z}, 0}$.

In all the examples so far discussed, the steady state $\widehat{E}_{\widehat{z}, 0}$ in which all agents adopt clean technology is better than the steady state $\bar{E}_{\bar{z}, 1}$ from an economic as well as ecological point of view. In these cases, a good environmental and economic policy would be to avoid converging to the "sub-optimal" equilibrium $\bar{E}_{\bar{z}, 1}$.

\footnotetext{
${ }^{12}$ We assume that the regulator's discount rate $\delta$ in C.1 is equal to the decay rate in 9
} 

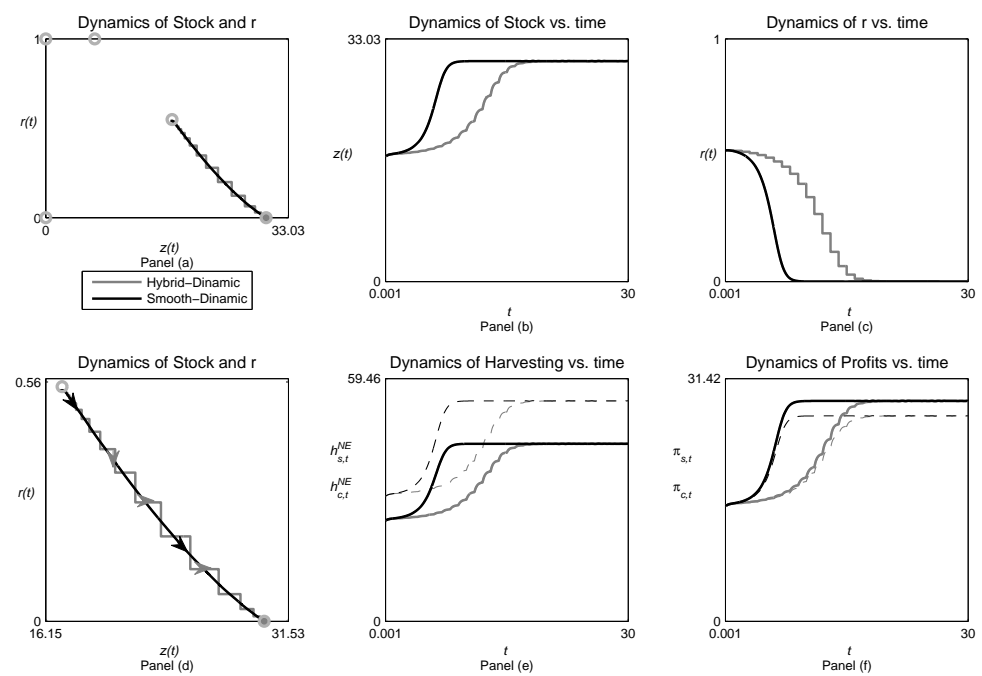

Figure 3: As Fig. 22 but with i.c. $(17,0.54)$.

As highlighted in the example of Figure (1), a higher propensity to pay for the product obtained through "clean" technology (e.g., the environmentally-friendly or the bycatch-free product, according to the interpretation given to the model), i.e., a higher $a_{c}$, could guarantee that a poverty trap equilibrium such as $\bar{E}_{\bar{z}, 1}$ is unstable, compare Panel (a) (where $a_{c}=1.45$ ) with Panel (b) (where $a_{c}=1.65$ ) of Figure (1). Therefore, to stimulate the adoption of "clean" technology, a possible solution would be to make consumers more willing to pay for the "clean" product ${ }^{13}$ This can be achieved in several ways, for instance through eco-labeling, tax reliefs or awarenessraising campaigns on environmental issues. However, if the propensity to pay for the "clean" product $a_{c}$ becomes too high, then other undesired effects could arise. In fact, the equilibrium $E_{\widehat{z}, 0}=\left(\frac{\alpha}{\beta}-\frac{N a_{c} q_{c}}{2 \beta \gamma}, 0\right)$ has a contact with the border equilibrium $E_{0,0}$ at $a_{c}=\frac{\alpha \beta \gamma}{N q_{c}}$ and it disappears from the positive orthant through a transcritical bifurcation for higher values of $a_{c}$. This situation is depicted in Figure (1), Panel (c), where the only stable equilibrium is the inner fixed point $E^{*}$. At this equilibrium, less than half of the exploiters employ the environmentally-friendly

\footnotetext{
${ }^{13}$ The dynamics of the hybrid model and the continuous one are similar for the case of Figure (1), Panel (b), and the dynamics of the hybrid model are not so sensitive to the value of the intensity of choice parameter $\theta$. Hence, for the sake of saving space the related simulations are omitted.
} 
technology and the level of the natural resource stock is relatively low; it is useful to compare the level of the resource stock for the stable equilibria in Figure (1), Panel (a) and Panel (b) with the one in Panel (c). This numerical example highlights a critical aspect of controlling the propensity to pay for the environmentally-friendly product as a possible tool to increase sustainability. This can be better understood by observing the dynamics of the profits and the harvests. Figure (4) shows the trajectories of the continuous-time and hybrid models for parameters as in Figure (1), Panel (c) and i.c. $(6,0.9)$. Under this parameter setting, it is $h_{c}^{N E}(z(t))>h_{s}^{N E}(z(t))$, i.e., any agent adopting strategy $c$ catches more than any agent adopting strategy $s$. This is due to the higher reservation price consumers have for buying an "environmentally-friendly" product. Thus, as the number of agents using the "environmentally-friendly" technology increases, the total biomass decreases. At a certain point, due to a reduction in the level of the stock and to the higher fixed cost of strategy $c$, agents find it more profitable to choose standard technology $s$ and switch to it. As a result, $\dot{r}$ becomes positive and the level of biomass starts increasing, till the point in which the environmentally-friendly strategy becomes more profitable again. In this example, the "environmentally-friendly" strategy $c$ is more profitable (and so $\dot{r}<0$ ) as long as the level of biomass is sufficiently high. This process explains intuitively the oscillatory convergence to the inner equilibrium $E^{*}$. Moreover, if consumers are too keen to pay for the product obtained with the "environmentally-friendly" technology and fishermen are myopic, deciding to harvest "Nash" quantities and to select the harvesting technology according to past observed profits, then the fishery may be characterized by overexploitation. In this case, agents would be better off using standard technology s. In fact, by (C.5) and (C.6), a regulator would impose the control $r=1$ from the beginning to maximize total revenues over time. Of course this consideration does not take into account other reasons for employing clean technology, such as bycatch reduction or pollution issues, but only considers the net economic benefit for fishermen. Explicit tracking of other environmental variables could be addressed in future extensions of this paper.

For that which concerns the similarities and differences between the hybrid and the continuoustime models, Figure (4) shows that the hybrid model with a low intensity of choice (here it is $\theta=0.5$ ) yields a transient dynamic that is very similar to the one in continuous-time. Both models converge to the stable inner equilibrium $E^{*}$ and all the previous considerations are still valid for the hybrid model. However, differently from the case of Figure (1), Panels (a) and (b), in the example of Figure (1), Panel (c), the dynamics of the hybrid model are quite sensitive to changes of the parameter $\theta$. 
For small values of $\theta$ (such as $\theta=0.05$ ), the inner equilibrium is still the only stable attractor for the hybrid model and we observe non-oscillatory convergence to it, see Figure (5). On the contrary, if $\theta$ is high enough, the inner equilibrium $E^{*}$ becomes unstable and the generic trajectory converges to a chaotic attractor. Further increasing the intensity of choice $\theta$, an attractor surrounding the unstable inner equilibrium $E^{*}$ coexists with the stable border equilibrium $\bar{E}_{\bar{z}, 1}$, see Figures 6 6 and (7) where $\theta=5$. Figure (6) displays a trajectory converging to the stable non-equilibrium attractor for i.c. $(6,0.5)$. Figure 77 exhibits a trajectory converging to the border equilibrium $\bar{E}_{\bar{z}, 1}$ for i.c. $(6,0.9)$. For a sufficiently high intensity of choice $\theta$, the border equilibrium $\bar{E}_{\bar{z}, 1}$ becomes the only stable attractor for the hybrid dynamical system (simulations are not reported here for saving space).

As recalled above, under these parameter values, the equilibrium $\bar{E}_{\bar{z}, 1}$ represents the optimal state for maximizing the total industry profits given the harvesting functions (4). Thus, for sufficiently high intensities of choice, myopic profit-driven fishermen can indeed reach this "optimal" equilibrium, after an irregular transient period (see again Figure (7)). On the contrary, if agents' intensity of choice is too low, the hybrid model will reach a sub-optimal outcome, converging either to the inner equilibrium $E^{*}$ or to a chaotic attractor.

In addition to the propensity to pay for the "clean" product, another parameter that can be easily controlled by a regulator is the difference between the fixed costs of the production of the two types of technologies, for instance through a fixed tax for operating with a specific technology. Below, we briefly analyze the effects of this difference on the dynamics of a model. Starting from the situation depicted in Figure (1), Panel (a), the fixed cost associated to clean technology $c_{c}$ is decreased (obviously the same can be obtained by increasing the cost of standard technology $c_{s}$ ), see Figure (8), Panel (b). The border equilibrium $\widehat{E}_{\widehat{z}, 0}$ does not change coordinates but it becomes the only stable equilibrium of the system so that in the end all the agents adopt the environmentallyfriendly technology, see Figure (8), Panel (c). If $c_{c}$ is increased instead of decreased, the results is that the equilibrium $\widehat{E}_{\widehat{z}, 0}$ becomes unstable and equilibrium $\bar{E}_{\bar{z}, 1}$ is the only stable one, see Figure (8), Panel (a). This example is a simple application of Proposition 1, and shows how fixed costs can be employed to guarantee the convergence for the desired equilibrium in the continuous-time model. Clearly, the difference between the fixed costs make it so that the cheapest technology is more appealing than the other without affecting the levels of harvesting, see equations (4).

From a "bioeconomic" point of view, these numerical examples underline two main aspects. 

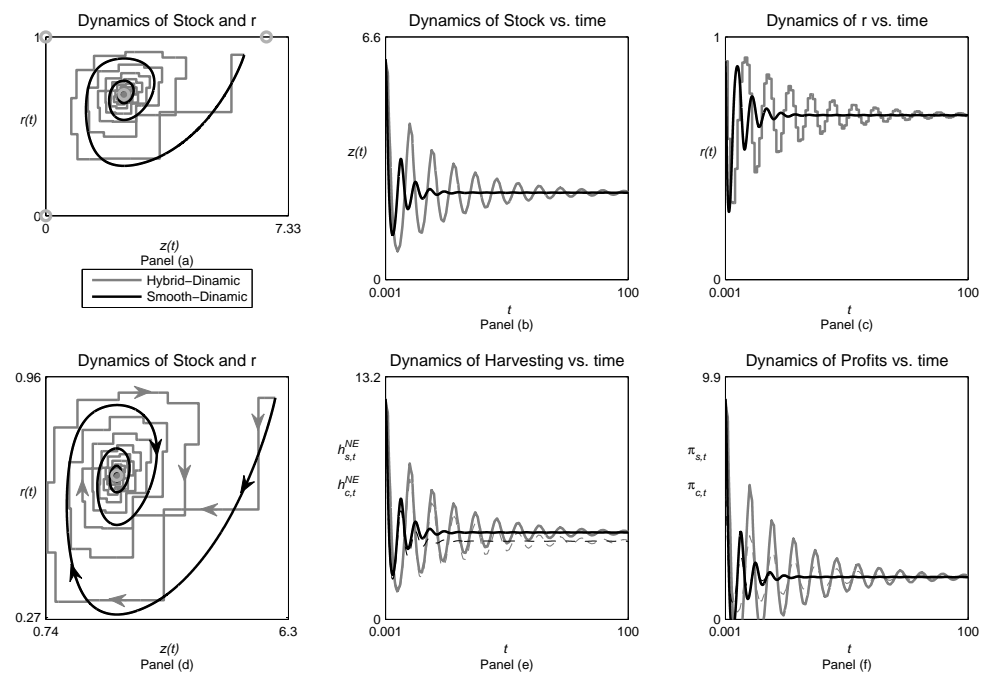

Figure 4: Dynamics of the model with continuous switchings (6) (black lines) and discrete switchings (gray lines) with parameter values as in Fig. (1), Panel (b), and with $\delta=0.01, \theta=0.5$ and i.c. $(6,0.9)$. Panels follow the setup of Fig. 22.
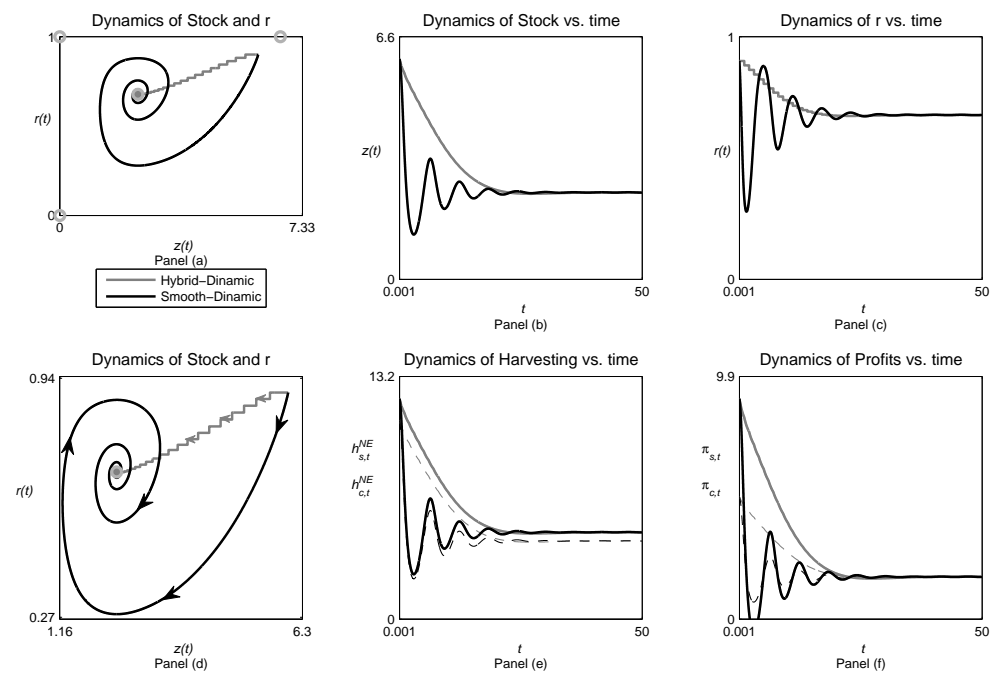

Figure 5: Dynamics of the model with continuous switchings 6) (black lines) and discrete switchings 10 (gray lines) with parameter values and i.c. as in Fig. (4). but $\theta=0.05$. Order of panels as in Fig. 22. 

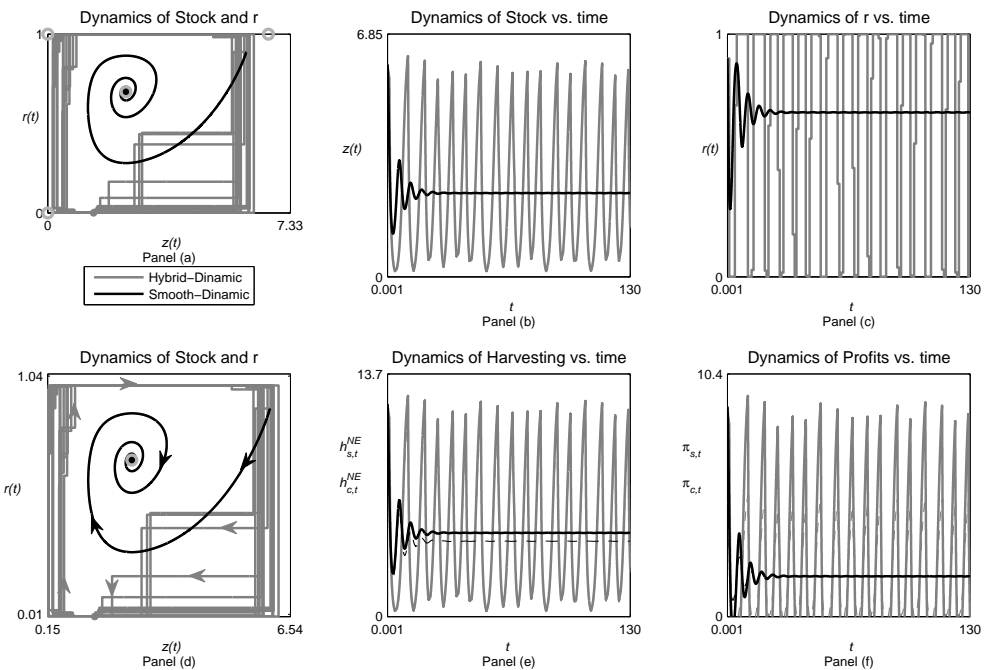

Figure 6: Dynamics of the model with continuous switchings 6) (black lines) and discrete switchings 10 (gray lines) with parameter values as in Fig. (4) but $\theta=5$ and i.c. $(6,0.9)$. Order of panels as in Fig. (2).
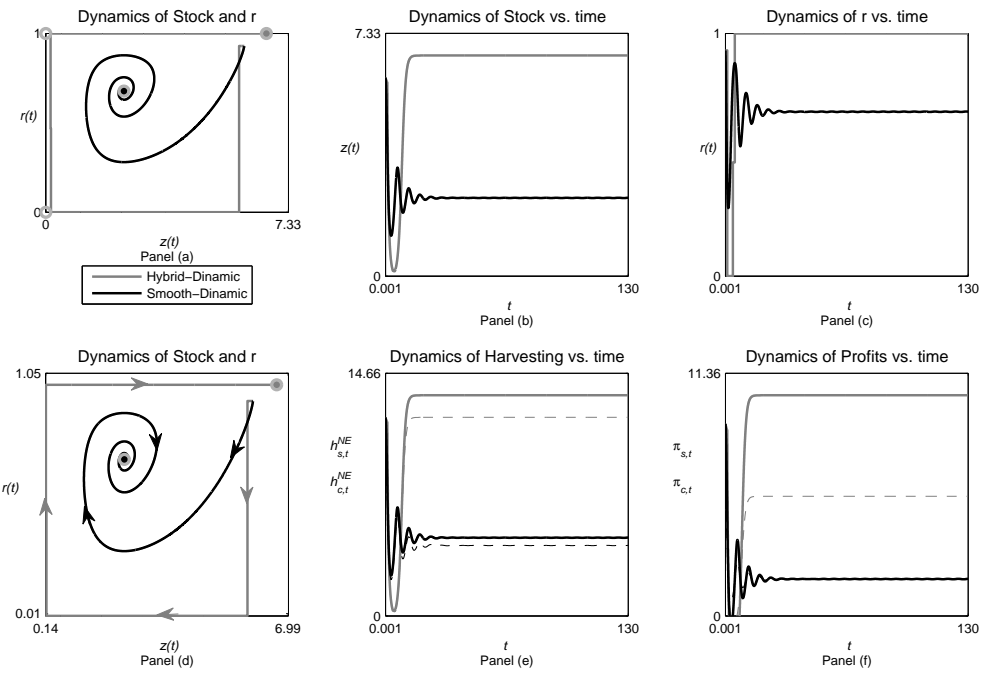

Figure 7: Dynamics of the model with continuous switchings (6) (black lines) and discrete switchings (gray lines) with parameter values as in Fig. 6). but $\theta=5$ and i.c. $(6,0.93)$. Order of panels as in Fig. 22. 

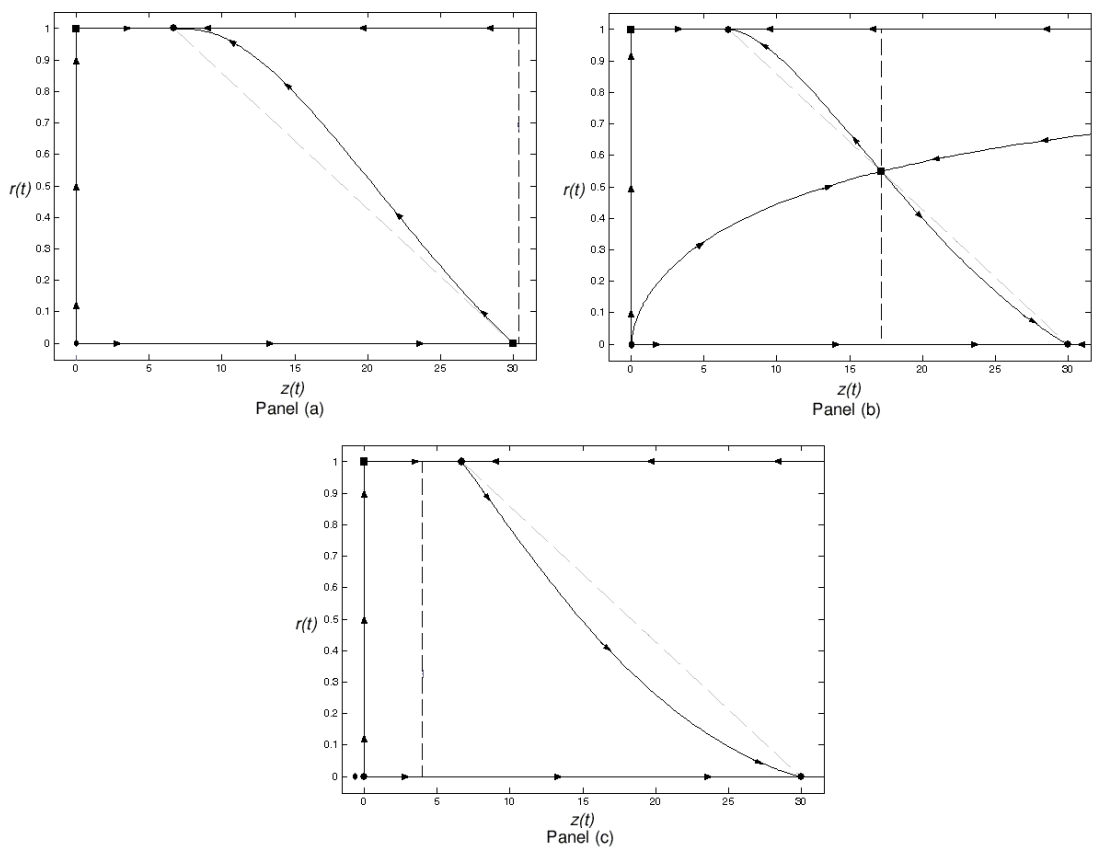

Figure 8: State spaces of the dynamical system (6). Panel (a): $\alpha=19, \beta=0.15, a_{s}=1, a_{c}=1.45, c_{s}=0.4, c_{c}=6$, $N=10, q_{s}=1.8, q_{c}=1, \gamma=0.5$; Panel (b): as in Panel (a) but $c_{c}=3$; Panel (c) as in Panel (a) but $c_{c}=1$. Black squares, circles and ovals represent, respectively, saddle points, stable nodes and unstable nodes. Dashed lines are the isoclines $\dot{z}=0$ (gray) and $\dot{r}=0$ (black). The black lines represent stable and unstable manifolds of the saddle points. The arrows indicate the direction of the flow of the dynamical system along the manifolds.

First, the gap between the efficiencies of the two technologies can lead to a resource overexploitation whenever this gap is more than compensated by the higher consumers' reservation price for an "environmentally-friendly" product. Second, lowering fixed costs for clean technology increases the fraction of agents selecting it without directly affecting the level of harvesting. Thus, fixed costs could be employed as a control variable to stimulate the adoption of clean technology.

Up to now, all the simulations presented refer to case $\xi=c_{c}-c_{s}>0$, i.e. where clean technology is characterized by higher fixed costs. In the opposite situation, i.e. for $\xi=c_{c}-c_{s}<0$, similar dynamics are obtained as indicated in Figure (9), in which three typical phase portraits of the model in continuous-time (6) are depicted for parameters: $\alpha=26, \beta=5, a_{s}=1, a_{c}=2, c_{s}=1$, $c_{c}=0.4\left(\right.$ Panel (a)), $0.5(\operatorname{Panel}(\mathrm{b}))$ and $0.6(\operatorname{Panel}(\mathrm{c})), N=10, q_{s}=1, q_{c}=0.185, \gamma=0.5$. Panel (a) shows a phase portrait with three saddle points $E_{0,0}, \widehat{E}_{\widehat{z}, 0}$, and $E_{0,1}$ and the stable focus 

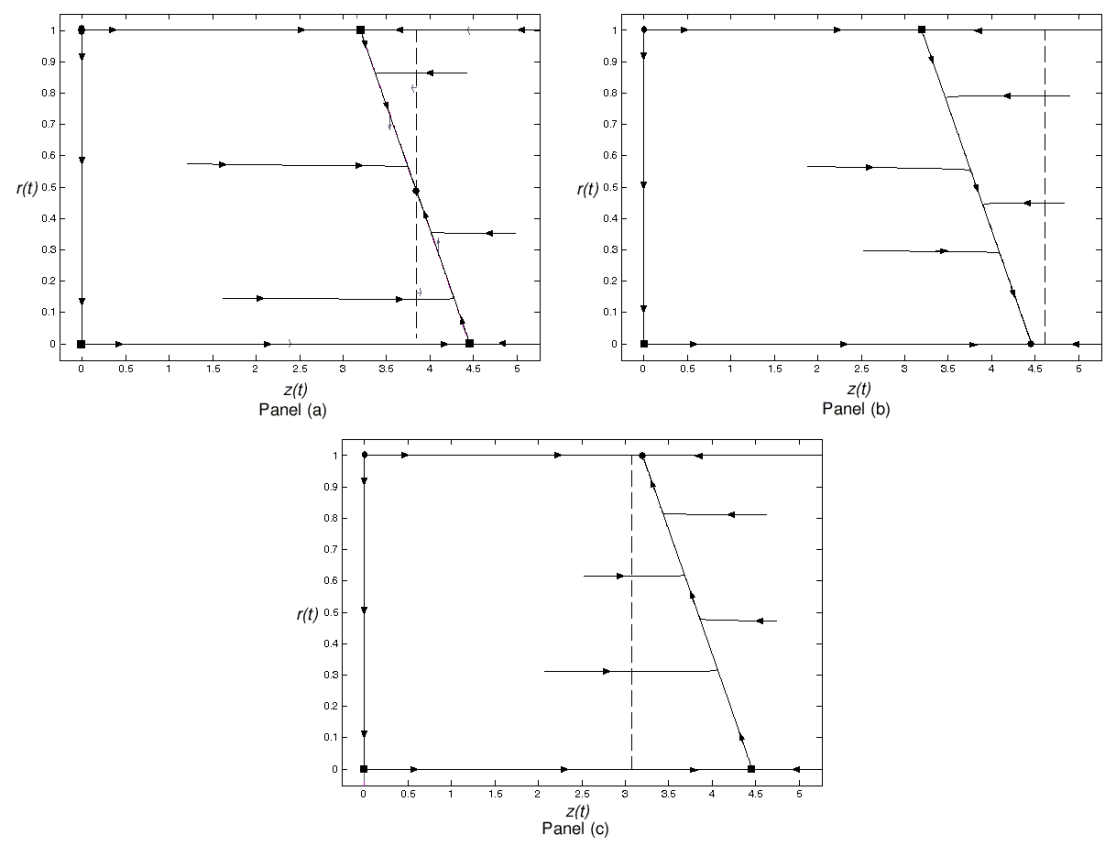

Figure 9: Phase portraits of the dynamical system 6). Panel (a) $\alpha=26, \beta=5, a_{s}=1, a_{c}=2, c_{s}=1, c_{c}=0.5$, $N=10, q_{s}=1, q_{c}=0.185, \gamma=0.5$; Panel (b) as in Panel (a) but $c_{c}=0.4$; Panel (c) as in Panel (a) but $c_{c}=0.6$. Black squares, circles and ovals represent, respectively, saddle points, stable nodes and unstable nodes. Dashed lines are the isoclines $\dot{z}=0$ (gray) and $\dot{r}=0$ (black). The black closed curve is an invariant orbit of the dynamical system and the black lines represent stable and unstable manifolds of the saddle points. The arrows indicate the direction of the flow of the dynamical system along the manifolds.

$E^{*}$. Under these parameters, an optimal control of the system (C.5) is achieved by fixing $r=0$. By decreasing the fixed cost of clean technology, it can indeed ensure that $\widehat{E}_{\widehat{z}, 0}$ is the only stable equilibrium under the evolutionary selection process, see Figure (9), Panel (b). On the contrary, if the fixed cost of clean technology is too high, the "sub-optimal" equilibrium $\bar{E}_{\bar{z}, 1}$ becomes the only stable one. Again, the difference in fixed costs is a key tool for driving the evolutionary process through the desired equilibrium.

In respect to the dynamics of the hybrid model with parameters as in Figure (9), Panel (b), no particular differences are present with the continuous-time model whenever the values of the intensity of choice are sufficiently small. In this case, differences can be observed only in the transient trajectories and so we do not report them here. On the contrary, when fishermen have a high propensity to switch between strategies according to their past-profit performances, the inner 

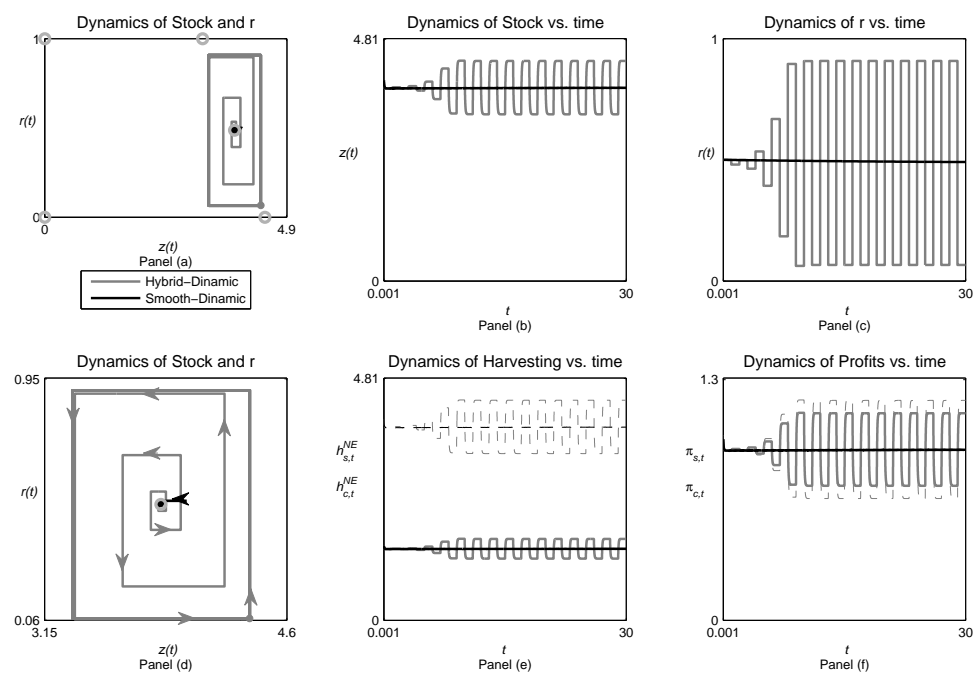

Figure 10: Dynamics of the model with continuous switchings 6 (black lines) and discrete switchings 10 (gray lines) with parameter values as in Fig. 99, Panel (a), and with $\theta=80$ and i.c. $(4,0.5)$. Order of panels as in Fig. (2).

equilibrium becomes unstable for the hybrid model and a cyclic attractor appears around it. Figure 10. represents such a case with $\theta=80$. Comparing Panels (b), (c), (d) and (e) in Figure (10), one can easily observe that the harvesting strategy $s$ provides a better performance when the level of fish biomass is relatively high. In fact, around the inner equilibrium the level of biomass is high enough to make strategy $s$ more profitable than strategy $c$. Given the relatively high intensity of choice, fishermen are sensitive to small differences of profits and most of them decide to adopt strategy $s$. This reduces the level of biomass due to higher harvesting implied by strategy $s$ in this specific case. With reduced harvesting, strategy $c$ procures a better economic performance due to its lower fixed costs (recall that here it is $\xi=c_{c}-c_{s}<0$ ). Fishermen react to this change and start to adopt strategy $c$. In this case, a short period with a high fraction of agents using technology $c$ helps to increase the level of biomass. As a consequence, strategy $s$ becomes more profitable again and cyclic dynamics persist in the long run. This example suggests that the presence of fishermen who are sensible to past performances could in some cases destabilize the dynamics.

In numerical simulation not reported here, the hybrid model exhibits similar dynamics to the 
continuous-time model for parameters as in Figure (9), Panels (a) and (c).

The numerical simulations discussed in this section point out two important aspects. First of all, the continuous-time model 6 and the hybrid model 10 show similar dynamics when the intensity of choice $\theta$ is low. On the contrary, when $\theta$ assumes larger values, overshooting can take place. Although all the simulations are made for a specific value of $\Delta l$ and $\delta$, a large number of numerical tests confirms that this holds true for a wide range of these parameters. The second important aspect concerns the bioeconomic implications of adopting "clean" technology. A high propensity to pay for the clean product increases the fraction of exploiters adopting it. However, if this propensity is too high, then it compensates for the reduction in harvesting due to the lower efficiency of clean technology and problems of overexploitation of the resource might arise. Last but not least, controlling the fixed costs of the two technologies could ensure the convergence to the desired equilibrium in the evolutionary system thus reducing the risks of overexploitation of the resource, as pointed out by several examples in this section.

\section{Conclusions}

In this paper we have studied a dynamical model of technology adoption for the exploitation of a renewable resource. Technology adoption is modeled through an evolutionary game, where agents can change strategies (i.e. the employed technology) continuously or at specified times. In the latter case the mathematical description of the system gives rise to a hybrid model. Similarities and differences between the two possible dynamical systems are outlined in this paper, mainly through numerical simulations. Continuous switching in general amplifies the final outcomes of the system in both negative and positive cases. Despite the fact that discrete-time replicator models are known to generate more complicated behaviors than those of a continuous-time (see, e.g. [35]), we showed several examples in which discrete switching may have a stabilizing effect: in fact, a form of inertia is introduced in the system when agents assess the fitness of each strategy according to the moving average of past profits over an interval of time. Overall, with continuous-time switching or with discrete-time switching and low switching propensity, the system is likely to be trapped in basins of non-optimal attractors. This occurs when other attractors of the system could guarantee more profits in the long-run. This comparison can be established by solving an intertemporal profit maximization problem with the same harvesting functions employed by the myopic agents. The numerical results suggest that the inner equilibrium of the hybrid model is destabilized whenever the 
corresponding inner equilibrium of the continuous model has complex conjugate eigenvalues with negative real part or for high enough values of the intensity of choice. Hence, when the equilibrium in the continuous model is a stable focus, the value of the intensity of choice in the hybrid model is likely to play a crucial role in the long-run dynamics of the system.

The results confirm pretty intuitive and common sense concepts in natural resource modeling as well as some aspects of the problem that are less intuitive. In most cases higher immediate profits entail less welfare in the long run, because of "resource externalities". A high willingness to pay for the "greener" product can indeed lead to over-harvesting and resource depletion, although the use of environmentally-friendly technology is prevalent. Our examples suggest that one does not only have to harvest differently, but also to harvest less. The market is not able to self-regulate in these delicate issues and, due to path dependence of the system, it is likely to be "locked-in" in sub-optimal outcomes. Bycatch-free labels could be an important example to follow, but it is also necessary to reduce total catches (at least in the short-run), to establish no-take zones (on this point see [17, [27] and [5]) and to integrate the control of exploitation activities with the dynamics of the whole ecosystem (reduction of bycatch and pollution). One possible extension of the model is to explicitly consider the bycatch dynamics and to study the condition for the sustainable exploitation of the target and non-target species under different assumptions on their relationship (predatorprey, symbiotic interaction or negative interaction). In addition, it would be interesting to study the evolutionary model with switching costs, as indicated in Appendix A.

\section{Acknowledgments}

The authors would like to thank the participants at the $7^{\text {th }}$ MDEF Workshop in Urbino, September 2012 and at the $19^{\text {th }}$ CEF Conference in Vancouver, July 2013, for helpful discussions as well as two anonymous referees for their detailed and insightful comments. The usual disclaimers apply.

This work has been performed within the activity of the PRIN project "Local interactions and global dynamics in economics and finance: models and tools", MIUR (Italian Ministry of Education, University and Research), and under the auspices of COST Action IS1104 "The EU in the new complex geography of economic systems: models, tools and policy evaluation". 


\section{Appendix A.}

Assuming the presence of switching costs, let us say $c_{1}$ for switching from technology $c$ to $s$ and $c_{2}$ for switching from technology $s$ to $c$, the dynamical system (5) takes the form:

$$
\left\{\begin{array}{l}
\dot{z}(t)=z(t)(\alpha-\beta z(t))-N\left(r(t) h_{s}^{N E}(z(t))+(1-r(t)) h_{c}^{N E}(z(t))\right) \\
\dot{r}(t)=r(t)(1-r(t))\left\{\begin{array}{lll}
{\left[\pi_{s}^{*}(z(t))-\pi_{c}^{*}(z(t))-c_{1}\right]} & \text { iff } & \pi_{s}^{*}(z(t))-\pi_{c}^{*}(z(t)) \geq c_{1}, \\
0 & \text { iff } & -c_{2}<\pi_{s}^{*}(z(t))-\pi_{c}^{*}(z(t))<c_{1} \\
{\left[\pi_{s}^{*}(z(t))-\pi_{c}^{*}(z(t))+c_{2}\right]} & \text { iff } & \pi_{s}^{*}(z(t))-\pi_{c}^{*}(z(t)) \leq-c_{2}
\end{array}\right.
\end{array}\right.
$$

see, e.g., 31. Model A.1 is a piecewise differentiable (or piecewise smooth) dynamical system in continuous time, so that its dynamic properties can be different from the one of model (5). For a general treatment of piecewise smooth dynamical systems see, e.g., [16] and [29, for an example of their application to economics, see, e.g., [4].

\section{Appendix B. Proof of Proposition 1}

Computing the Jacobian matrix of the ODEs system (6) we get

$$
J(z, r)=\left(\begin{array}{cc}
\frac{-N a_{c} q_{c}(1-r)-N a_{s} q_{s} r+2(\alpha-2 z \beta) \gamma}{2 \gamma} & \frac{\left(a_{c} q_{c}-a_{s} q_{s}\right) z N}{2 \gamma} \\
-\frac{\left(a_{c}^{2} q_{c}-a_{s}^{2} q_{s}\right)(1-r) r}{4 \gamma} & \frac{(-1+2 r)\left(a_{c}^{2} q_{c} z-a_{s}^{2} q_{s} z-4 \xi \gamma\right)}{4 \gamma}
\end{array}\right)
$$

In the equilibrium $(0,0)$ it is

$$
J(0,0)=\left(\begin{array}{cc}
\alpha-\frac{N a_{c} q_{c}}{2 \gamma} & 0 \\
0 & \xi
\end{array}\right)
$$

so that, apart from the eigenvalue $\xi,(0,0)$ is unstable with eigenvalue $\alpha-\frac{N a_{c} q_{c}}{2 \gamma}>0 \Leftrightarrow a_{c} q_{c}<$ $\frac{2 \gamma \alpha}{N} \Leftrightarrow \widehat{E}_{\widehat{z}, 0}>0$.

For the stability in $\widehat{E}_{\widehat{z}, 0}=\left(\frac{2 \alpha \gamma-N a_{c} q_{c}}{2 \beta \gamma}, 0\right)$, it is

$$
J\left(\widehat{E}_{\widehat{z}, 0}\right)=\left(\begin{array}{cc}
-\alpha+\frac{N a_{c} q_{c}}{2 \gamma} & -\frac{N\left(a_{c} q_{c}-a_{s} q_{s}\right)\left(N a_{c} q_{c}-2 \alpha \gamma\right)}{4 \beta \gamma^{2}} \\
0 & \frac{\left(N a_{c} q_{c}-2 \alpha \gamma\right)\left(a_{c}^{2} q_{c}-a_{s}^{2} q_{s}\right)}{8 \beta \gamma^{2}}+\xi
\end{array}\right)
$$

and condition $\widehat{E}_{\widehat{z}, 0}>0 \Leftrightarrow-\alpha+\frac{N a_{c} q_{c}}{2 \gamma}<0$. Thus, $\widehat{E}_{\widehat{z}, 0}>0$ is stable also along the second eigendirection if $\xi<\widehat{\xi}$. If the reverse inequality holds then $\widehat{E}_{\widehat{z}, 0}$ is a saddle. The stability in $(0,1)$ 
and in $\bar{E}_{\bar{z}, 1}$ is studied analogously. Notice that if $\xi<0$ then $\widehat{E}_{\widehat{z}, 0}$ and $\bar{E}_{\bar{z}, 1}$ cannot be stable at the same time. On the contrary, if $\xi>0$ then $\widehat{E}_{\bar{z}, 0}$ and $\bar{E}_{\bar{z}, 1}$ can be stable at the same time. This case occurs if $\widehat{E}_{\widehat{z}, 0}$ and $\bar{E}_{\bar{z}, 1}$ are both stable, i.e. $\bar{\xi}<\xi<\widehat{\xi}$.

Now we consider the inner equilibrium $E^{*}=\left(z^{*}, r^{*}\right)$

$$
J\left(z^{*}, r^{*}\right)=\left(\begin{array}{cc}
-\frac{4 \xi \beta \gamma}{a_{c}^{2} q_{c} a_{s}^{2} q_{s}} & \frac{2 \xi N\left(a_{c} q_{c}-a_{s} q_{s}\right)}{a_{c}^{2} q_{c}-a_{s}^{2} q_{s}} \\
\frac{-\left[\left(N a_{c} q_{c}-2 \alpha \gamma\right)\left(a_{c}^{2} q_{c}-a_{s}^{2} q_{s}\right)+8 \xi \beta \gamma^{2}\right]\left[\left(N a_{s} q_{s}-2 \alpha \gamma\right)\left(a_{s}^{2} q_{s}-a_{c}^{2} q_{c}\right)-8 \xi \beta \gamma^{2}\right]}{4 N^{2} \gamma\left(a_{c} q_{c}-a_{s} q_{s}\right)^{2}\left(a_{c}^{2} q_{c}-a_{s}^{2} q_{s}\right)} & 0
\end{array}\right)
$$

assuming positive biomass at the equilibrium $z^{*}=\frac{4\left(c_{c}-c_{s}\right) \gamma}{a_{c}^{2} q_{c}-a_{s}^{2} q_{s}}>0$, the Jacobian has the form $J\left(z^{*}, r^{*}\right)=\left(\begin{array}{cc}J_{11}<0 & J_{12} \\ J_{21} & 0\end{array}\right)$, with characteristic polynomial $\lambda^{2}-J_{11} \lambda-J_{12} J_{21}=0$. From the fact that $\operatorname{Trace}(J)<0$ we deduce that $E^{*}$ is stable (either a spiral or a node) whenever $J_{12} J_{21}<0$, i.e. $\widehat{\xi}<\xi<\bar{\xi}$, or a saddle point whenever $J_{12} J_{21}>0$, i.e. $\bar{\xi}<\xi<\widehat{\xi}$.

To sum up: (i) in the case $\widehat{\xi}<\xi<\bar{\xi}$, $E^{*}$ is stable (spiral or node) with saddle points $\widehat{E}_{\widehat{z}, 0}$ and $\bar{E}_{\bar{z}, 1}$; (ii) in the case $\bar{\xi}<\xi<\widehat{\xi}, E^{*}$ is a saddle with stable nodes $\widehat{E}_{\widehat{z}, 0}$ and $\bar{E}_{\bar{z}, 1}$; (iii) in the other cases $E^{*}$ is unfeasible. Moreover: (iv) at $\xi=\widehat{\xi}(\xi=\bar{\xi})$ a transcritical bifurcation occurs at which $E^{*}$ and $\widehat{E}_{\widehat{z}, 0}$ (respectively $E^{*}$ and $\bar{E}_{\bar{z}, 1}$ ) exchange their stability properties.

\section{Appendix C. Optimal Control of fishing technologies}

Assume that agents employ the Nash strategies (4) but they agree to fix optimally $r$ (the fraction of agents using the "standard" technology) in order to maximize total industry profits. Otherwise, we could interpret this case as if a regulator imposes the share of agent employing the standard technology in order to maximize intertemporal profits, letting agents adopt myopic harvesting strategies (4). The regulator's problem is

$$
\begin{aligned}
& \max _{r(t) \in[0,1]} \int_{0}^{+\infty} e^{-\delta t}\left[\left(r(t)\left(\frac{a_{s}^{2} q_{s}}{4 \gamma} z(t)-c_{s}\right)+(1-r(t))\left(\frac{a_{c}^{2} q_{c}}{4 \gamma} z(t)-c_{c}\right)\right)\right] d t \\
& \text { s.t. } \\
& \dot{z}=G(z(t))-N\left[r(t) \frac{a_{s} q_{s}}{2 \gamma} z(t)+(1-r(t)) \frac{a_{c} q_{c}}{2 \gamma} z(t)\right] \\
& z(t) \geq 0
\end{aligned}
$$

where $\delta$ is the instantaneous discount rate. 
The current value Hamiltonian function is linear in the control $r$ :

$$
H=\frac{1}{4 \gamma}\left(4 \lambda \gamma G(z)-4 c_{c} \gamma+a_{c}^{2} q_{c} z-2 N a_{c} q_{c} \lambda z+p(\lambda, z) \cdot r\right)
$$

where the switching function $p(\lambda, z)$ is given by

$$
p(\lambda, z)=4 \gamma\left(c_{c}-c_{s}\right)-z\left(a_{c}^{2} q_{c}-a_{s}^{2} q_{s}\right)+2 N \lambda z\left(a_{c} q_{c}-a_{s} q_{s}\right)
$$

The optimal control is either 'bang-bang' or singular:

$$
r^{*}=\left\{\begin{array}{l}
0 \text { if } p(\lambda, z)<0 \\
\text { singular if } p(\lambda, z)=0 \\
1 \text { if } p(\lambda, z)>0
\end{array}\right.
$$

If $p(\lambda, z)=0$ over an interval $\left[t_{1}, t_{2}\right]$, then a singular control is required. The singular steady state can be determined according to [13. At a steady state $\dot{z}=\dot{\lambda}=0$, the following modified 'golden-rule' condition holds

$$
\delta=G^{\prime}\left(z^{*}\right)-\frac{N a_{c} a_{s} q_{c} q_{s}\left(a_{c}-a_{s}\right) z^{* 2}-8\left(c_{c}-c_{s}\right) \gamma^{2} G\left(z^{*}\right)}{2 \gamma z^{*}\left(\left(a_{c}^{2} q_{c}-a_{s}^{2} q_{s}\right) z^{*}-4 \gamma\left(c_{c}-c_{s}\right)\right)}
$$

Assuming $G(z)=z(\alpha-\beta z)$, we can calculate numerically (or analytically) a unique singular steady state $z^{*}$. The optimal singular control $r^{*}$ can be then determined by substituting $z^{*}$ in the motion equation with $\dot{z}=0$, i.e.

$$
r^{*}=\frac{N a_{c} q_{c}-2 \gamma\left(\alpha-\beta z^{*}\right)}{N\left(a_{c} q_{c}-a_{s} q_{s}\right)}
$$

For such a problem the optimal harvest strategy consists in bang-bang control only or in the most rapid approach to the equilibrium $z^{*}$ through some combination of bang-bang controls and singular control, see [13] for further discussions on the topic.

\section{References}

[1] A. Antoci, R. Dei, and M. Galeotti. Financing the adoption of environment preserving technologies via innovative financial instruments: An evolutionary game approach. Nonlinear Analysis: Theory, Methods 83 Applications, 71(12):e952 - e959, 2009.

[2] A. Antoci, M. Galeotti, and D. Radi. Financial tools for the abatement of traffic congestion: A dynamical analysis. Computational Economics, 38:389-405, 2011. 
[3] J. P. Aubin, J. Lygeros, M. Quincampoix, S. S. Sastry, and N. Seube. Impulse differential inclusions: a viability approach to hybrid systems. IEEE Transactions on Automatic Control, $47(1): 2-20,2002$.

[4] G. I. Bischi, C. Chiarella, M. Kopel, and F. Szidarovszky. Nonlinear Oligopolies: Stability and Bifurcations. Springer-Verlag, 2010.

[5] G. I. Bischi and F. Lamantia. Harvesting dynamics with protected and unprotected areas. Journal of Economic Behavior and Organization, 62:348-370, 2007.

[6] G. I. Bischi, F. Lamantia, and D. Radi. Multi-species exploitation with evolutionary switching of harvesting strategies. Natural Resource Modeling, In press, 2013.

[7] G. I. Bischi, F. Lamantia, and D. Radi. A preypredator fishery model with endogenous switching of harvesting strategy. Applied Mathematics and Computation, 219(20):10123-10142, 2013.

[8] G. I. Bischi, F. Lamantia, and L. Sbragia. Competition and cooperation in natural resources exploitation: an evolutionary game approach. In: Carraro, C., Fragnelli, V. (Eds.), Game Practice and the Environment, Edward Elgar Publishing edition, 2004.

[9] G. I. Bischi, F. Lamantia, and L. Sbragia. Strategic interaction and imitation dynamics in patch differentiated exploitation of fisheries. Ecological Complexity, 6:353-362, 2009.

[10] G. I. Bischi and D. Radi. An extension of the Antoci-Dei-Galeotti evolutionary model for environment protection through financial instruments. Nonlinear Analysis: Real World Applications, 13(1):432-440, 2012.

[11] M. S. Branicky. Stability of switched and hybrid systems. American Control Conference, 4:3498-3503, 1994.

[12] M. S. Branicky. Stability of hybrid systems: State of the art. Proc. IEEE Conf. on Decision and Control, San Diego, CA, pages 120-125, 1997.

[13] C. W. Clark. Mathematical bioeconomics: The optimal management of renewable resources. Wiley-Intersciences, New-York, 2nd edition edition, 1990.

[14] H. Dawid. Handbook of Computational Economics, volume 2. Edited by Leigh Tesfatsion and Kenneth L. Judd. Elsevier B. V., 2006. 
[15] F. Dercole, C. Prieu, and S. Rinaldi. Technological change and fisheries sustainability: The point of view of adaptive dynamics. Ecological Modelling, 379-387(3):379-387, 2010.

[16] M. Di Bernardo, M. I. Feigin, S. J. Hogan, and M. E. Homer. Local analysis of $C$-bifurcations in n-dimensional piecewise-smooth dynamical systems. Chaos, Solitons $\& 3$ Fractals, 10(11):1881$1908,1999$.

[17] B. Dubey, Peeyush Chandra, and Prawal Sinha. A model for fishery resource with reserve area. Nonlinear Analysis: Real World Applications, 4(4):625-637, 2003.

[18] S. Gao, D. Xie, and L. Chen. Pulse vaccination strategy in a delayed SIR epidemic model with vertical transmission. Discrete and continuous dynamical systems-Series B, 7(1):1531-3492, 2007.

[19] R. Goebel, R. G. Sanfelice, and A. R. Teel. Hybrid dynamical systems. Control Systems Magazine, 2:28-93, 2009.

[20] W. M. Haddad, V. Chellaboina, and S. G. Nersesov. Impulsive and Hybrid Dynamical Systems: Stability, Dissipativity, and Control. Princeton University Press, 2006.

[21] G. Hardin. The tragedy of the commons. Science, 162:1243-1248, December 1968.

[22] X-Z. He and K. Li. Heterogeneous beliefs and adaptive behaviour in a continuous-time asset price model. Journal of Economic Dynamics and Control, 36:973-987, 2012.

[23] R. L. Hicks and K. E. Schnier. Eco-labeling and dolphin avoidance: A dynamic model of tuna fishing in the eastern tropical pacific. Journal of Environmental Economics and Management, 2(56):103-116, 2008.

[24] J. Hofbauer and K. Sigmund. Evolutionary Games and Population Dynamics. Cambridge University Press, Beverly Hills CA, 1998.

[25] J. Hofbauer and K. Sigmund. Evolutionary game dynamics. Bulletin (New Series) of the American Mathematical Society, 40(4):479-519, 2003.

[26] C. Hommes. Bounded Rationality and Learning in Complex Markets. Handbook of Economic Complexity, Edited by J. Barkley Rosse, Jr., Cheltenham: Edward Elgar, 2009. 
[27] F. Lamantia. Variable effort management of renewable natural resources. Chaos, Solitons $\&$ Fractals, 29(3):771-782, 2006.

[28] D. Levhari and L. J. Mirman. The great fish war: An example using a dynamic Cournot-Nash solution. The Bell Journal of Economics, 11:322-334, 1980.

[29] E. Mosekilde and Z.T. Zhusubaliyev. Bifurcations and chaos in piecewise-smooth dynamical systems. World Scientific, 2003.

[30] J. Noailly, J. CJM van den Bergh, and C. A. Withagen. Evolution of harvesting strategies: replicator and resource dynamics. Journal of Evolutionary Economics, 13(2):183-200, 2003.

[31] T. P. Norman. The evolution of conflict under inertia. Working Paper (Nuffield College, Oxford), 2003.

[32] R. Sethi and E. Somanathan. The evolution of social norms in common property resource use. The American Economic Review, 86:766-788, 1996.

[33] M.F. Teisl, B. Roe, and R.L. Hicks. Can eco-labels tune a market? evidence from dolphin-safe labeling. Journal of Environmental Economics and Management, 43:339-359, 2002.

[34] J. Weibull. Evolutionary Game Theory. Cambridge, MA: The M.I.T. Press, 1995.

[35] F. J. Weissing. Evolutionary stability and dynamic stability in a class of evolutionary normal form games. Springer, In R. Selten (Ed.), Game Equilibrium Models I: Evolution and Game Dynamics edition, 1991.

[36] A. Xepapadeas. Regulation and evolution of compliance in common pool resources. Scandinavian Journal of Economics, 107(3):583-599, 2005. 